\title{
UNIQUENESS OF MAXIMAL ENTROPY ODD ORBIT TYPES
}

\author{
WILLIAM GELLER AND BENJAMIN WEISS
}

(Communicated by Barbara Lee Keyfitz)

\begin{abstract}
We prove that the maximal entropy orbit types of odd period for interval maps are unique. In fact we prove that they are uniquely maximal among all (not necessarily cyclic) permutations.
\end{abstract}

\section{INTRODUCTION}

Periodic orbits of interval maps can be classified combinatorially using the order inherited from the interval. For a continuous map, the existence of a periodic orbit of a given type typically entails the existence of many other orbit types and implies a lower bound on the topological entropy of the map. Several authors have studied the implications among orbit types and the relation between orbit types and entropy, including for example [Be, BCop, BGMY, GT, $\mathrm{J}]$ and the systematic treatment of [MN].

If $p_{1}<\cdots<p_{n}$ is any periodic orbit of a (continuous) map $f$ of a compact interval, we define the type of this orbit as the cyclic permutation $\theta$ on $\{1, \ldots, n\}$ given by $\theta(i):=j$ if $f\left(p_{i}\right)=p_{j}, 1 \leq i \leq n$. More generally, if $S=\left\{p_{1}, \ldots, p_{n}\right\}, p_{1}<\cdots<p_{n}$, and $f(S)=S$, we define the type of the finite invariant set $S$ to be the permutation $\theta$ given as before by $\theta(i):=j$ if $f\left(p_{i}\right)=p_{j}$.

We write $C_{n}$ for the set of all possible orbit types, or cycles, of period $n$, and $P_{n}$ for the set of all permutations on $\{1, \ldots, n\}$, and set $C:=\bigcup_{n \geq 1} C_{n}$, $P:=\bigcup_{n \geq 1} P_{n}$.

The dual of a permutation $\theta \in P_{n}$ is the permutation $\bar{\theta} \in P_{n}, \bar{\theta}(i)=$ $n+1-\theta(n+1-i)$, so that $\bar{\theta}$ is $\theta$ conjugated by a reversal of orientation.

Definition $1[\mathrm{MN}]$. The entropy of an orbit type $\theta \in C$ is

$$
h(\theta):=\inf \{h(f): f \text { is a map with an orbit of type } \theta\} .
$$

Here $h(f)$ is the topological entropy of the map $f$. Since the topological entropy of a map represents its dynamical complexity in an appropriate sense, the entropy of an orbit type $\theta$ can be thought of as representing the dynamical complexity required for any map with a periodic orbit of type $\theta$.

Received by the editors September 9, 1993.

1991 Mathematics Subject Classification. Primary 58F08, 54H20.

(C) 1995 American Mathematical Society $0002-9939 / 95 \$ 1.00+\$ .25$ per page 
More generally, if $\theta \in P$, we define $h(\theta):=\inf \{h(f): f$ has an invariant set of type $\theta$. It is easy to see that for all $\theta \in P, h(\bar{\theta})=h(\theta)$.

A permutation $\theta \in P$ forces $\eta \in P$ if every map having an invariant set of type $\theta$ also has one of type $\eta$.

In order to avoid handling the trivial case $n=1$, we establish the convention that " $n$ odd" will mean " $n>1$ odd" for the remainder of the paper.

For $n$ odd, define $l:=\lfloor(n-1) / 4\rfloor$ so that if $n \equiv 1(\bmod 4)$, then $n=4 l+1$ and if $n \equiv 3(\bmod 4)$, then $n=4 l+3$. Define the orbit type $\theta_{n}$ of period $n$ by

$$
j \mapsto \begin{cases}n-2 l-j & \text { if } 1 \leq j<n-2 l \text { and } j \text { odd } \\ j-n+2 l+1 & \text { if } n-2 l \leq j \leq n \text { and } j \text { odd } \\ n-2 l+j-1 & \text { if } 1 \leq j \leq 2 l \text { and } j \text { even } \\ n+2 l-j+2 & \text { if } 2 l<j \leq n \text { and } j \text { even }\end{cases}
$$

It can easily be verified that the $\theta_{n}$ are in fact cycles.

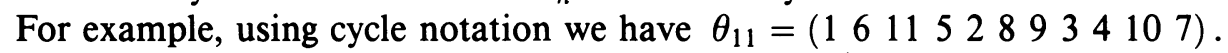
Misiurewicz and Nitecki [MN] first considered these orbit types, with $n \equiv 1$ $(\bmod 4)$.

It was proved in [GT] that the orbit types $\theta_{n}$ have maximal entropy among all $n$-cycles, and in fact among all $n$-permutations:

Theorem 1. For $n$ odd, $h\left(\theta_{n}\right)=\max \left\{h(\theta): \theta \in P_{n}\right\}$.

We extend here the methods of [GT] to prove these orbit types are the unique maximal entropy odd types (up to duality).

Theorem 2. For $n$ odd, if $\eta \in P_{n}$ and $h(\eta)=\max \left\{h(\theta): \theta \in P_{n}\right\}$, then $\eta=\theta_{n}$ or $\eta=\bar{\theta}_{n}$.

When $n$ is even, the maximal entropy cycles are not known. However, it is known that the maximal entropy permutations are not cycles, and come in self-dual pairs, in contrast to the situation for $n$ odd. A forthcoming paper of the first author and Zhang will address this.

\section{UNIQUENESS}

Proof of Theorem 2. For $\theta \in P_{n}$, define the primitive function $f_{\theta}$ on the interval $[1, n]$ as the piecewise linear interpolation of $\theta$. Define $M(\theta)$ to be the $(n-1)$ by $(n-1)$ matrix whose $(i, j)$ th entry is 1 if $f_{\theta}([i, i+1]) \supset[j, j+1]$ and 0 otherwise. It is well known [BCop] that for $\theta \in P, h(\theta)=\log \lambda$ where $\lambda$ is the spectral radius of $M(\theta)$.

For odd $n$, let $\eta \in P_{n}$ have maximal entropy, i.e., $h(\eta)=\max \{h(\theta): \theta \in$ $\left.P_{n}\right\}$. We need to show that $\eta=\theta_{n}$ or $\eta=\bar{\theta}_{n}$. Note that by the remarks preceding Theorem 11.6 in [MN], $\eta$ will not be forced by any other element of $P_{n}$ and $f_{\eta}$ will be maximodal and have all maximum values above all minimum values. Here $f_{\eta}$ maximodal means that it has a local extremum at $1,2, \ldots, n \in[1, n]$. Since $n$ is odd and $\eta$ is maximodal, either $\eta$ or $\bar{\eta}$ is normalized, i.e., either $f_{\eta}$ or $f_{\bar{\eta}}$ has a local minimum at $1 \in[1, n]$. Without loss of generality, assume $\eta$ is normalized. Then we show that $\eta=\theta_{n}$.

Let $r A=\lambda r$, where $A=M\left(\theta_{n}\right)$ and $h\left(\theta_{n}\right)=\log \lambda$, so that $r$ is a (left) Perron-Frobenius eigenvector for $A$ and $\lambda$ is the spectral radius of $A$. Set $B=M(\eta)$ so that $\lambda$ is also the spectral radius of $B$. 
Let $\varepsilon>0$. We define the matrix $B^{\varepsilon}=\left(B_{i j}^{\varepsilon}\right)$ by

$$
B_{i j}^{\varepsilon}=B_{i j}+\varepsilon\left[1-\prod_{i=1}^{n-1} \delta\left(A_{i j}, B_{i j}\right)\right],
$$

where $\delta(\cdot, \cdot)$ is Kronecker's delta. In other words, $B_{i j}^{\varepsilon}=B_{i j}+\varepsilon$ if the $j$ th column of $B$ differs from the $j$ th column of $A$, and otherwise $B_{i j}^{\varepsilon}=B_{i j}$.

Then $B^{\varepsilon}$ is irreducible.

This is true because $B^{\varepsilon}$ has a positive entry wherever $A$ has a positive entry, i.e., if $A_{i j}>0$, then $B_{i j}^{\varepsilon}>0$, since either $B_{i j}^{\varepsilon}=B_{i j}=A_{i j}>0$ or $B_{i j}^{\varepsilon}=B_{i j}+\varepsilon \geq \varepsilon>0$. But we will see in the remark following Proposition 1 that $A$ is irreducible; therefore $B^{\varepsilon}$ is also irreducible.

Let $k=(n-1) / 2$. Consider the Euclidean space $R^{n-1}=R^{2 k}$. A vector $w$ in this space will be denoted for notational convenience as

$$
w=(u, v)=\left(u_{1}, \ldots, u_{k}, v_{k}, \ldots, v_{1}\right) .
$$

At this point we need to introduce a cone, first defined in [GT], which will enable us to obtain more information about the relation between $A$ and $B$.

Definition 2. We say that $w$ belongs to the class of vectors $\mathscr{P} \subset R_{+}^{2 k}=\{x \in$ $\left.R^{2 k}: x \geq 0\right\}$ if the following two conditions hold:

(1.e) $v_{k} \geq u_{k} \geq u_{k-1} \geq v_{k-1} \geq v_{k-2} \geq \cdots \geq u_{2} \geq u_{1} \geq v_{1}$, if $k$ is even (i.e., if $n \equiv 1(\bmod 4))$.

(1.o) $v_{k} \geq u_{k} \geq u_{k-1} \geq v_{k-1} \geq v_{k-2} \geq \cdots \geq v_{2} \geq v_{1} \geq u_{1}$, if $k$ is odd (i.e., if $n \equiv 3(\bmod 4))$.

(2) $v_{k}-u_{k} \geq u_{k-1}-v_{k-1} \geq v_{k-2}-u_{k-2} \geq \cdots \geq(-1)^{k}\left(v_{1}-v_{1}\right) \geq 0$.

Note that $\mathscr{P}$ is a closed subcone of the positive cone.

As we will see in Lemma 1 , the eigenvector $r$ is in the interior of $\mathscr{P}$, so there exists $\varepsilon>0$ sufficiently small so that $r A \geq r B^{\varepsilon}$; this follows from applying the arguments of the proof of Proposition 10 in [GT] to those columns $B^{(j)}$ of $B$ which differ from the corresponding column $A^{(j)}$ of $A$ (exchanging strict for weak inequalities where necessary). Now let $\lambda_{\varepsilon}$ be the spectral radius of $B^{\varepsilon}$, and let $s_{\varepsilon}$ be a right Perron-Frobenius eigenvector: $B^{\varepsilon} s_{\varepsilon}=\lambda_{\varepsilon} s_{\varepsilon}$. Then

$$
\lambda\left(r, s_{\varepsilon}\right)=\left(\lambda r, s_{\varepsilon}\right)=\left(r A, s_{\varepsilon}\right) \geq\left(r B^{\varepsilon}, s_{\varepsilon}\right)=\left(r, B^{\varepsilon} s_{\varepsilon}\right)=\lambda_{\varepsilon}\left(r, s_{\varepsilon}\right) .
$$

This implies that $\lambda \geq \lambda_{\varepsilon}$ since $r$ is nonnegative and nonzero and $s_{\varepsilon}$ is positive by Perron-Frobenius and the irreducibility of $B^{\varepsilon}$.

But since $B^{\varepsilon}$ is irreducible and $B^{\varepsilon} \geq B$, we would have $\lambda_{\varepsilon}>\lambda$ unless $B^{\varepsilon}=B$. So $B^{\varepsilon}=B=A$ and we are finished.

Lemma 1. If $r$ is a (left) Perron-Frobenius eigenvector for $A=M\left(\theta_{n}\right)$, then it lies in the interior of $\mathscr{P}$.

That $r$ lies in $\mathscr{P}$ follows easily from the invariance of $\mathscr{P}$ under the action of $A^{2}$, proved in [GT]. For the proof of Theorem 2, it is crucial that $r$ is not on the boundary of the cone $\mathscr{P}$.

The proof of Lemma 1 relies on Lemma 2. 
Lemma 2. Let $n$ be odd, and let $A=M\left(\theta_{n}\right)$. If $n \equiv 3(\bmod 4)$, then

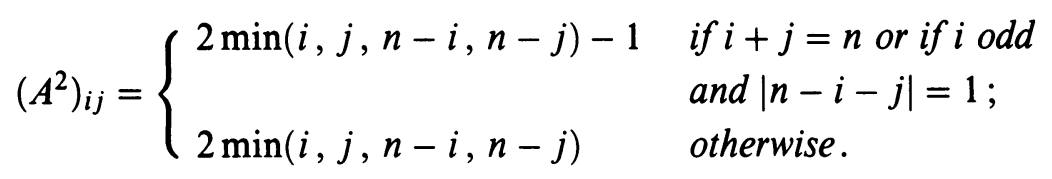

If $n \equiv 1(\bmod 4)$, then

$$
\left(A^{2}\right)_{i j}= \begin{cases}2 \min (i, j, n-i, n-j)-1 & \text { if } i+j=n \text { or if } i \text { even } \\ & \text { and }|n-i-j|=1 \\ 2 \min (i, j, n-i, n-j) & \text { otherwise. }\end{cases}
$$

Lemma 2 can be obtained by a routine calculation, which we omit, from the following fact, found in [GT]:

Proposition 1. Let $n$ be odd, $k=(n-1) / 2$, and $A=M\left(\theta_{n}\right)$. If $k$ is odd, then

$$
A_{i j}=1 \text { iff } \begin{cases}k-j+2 \leq i \leq k+j+1 & \text { for odd } j \leq k-2 \\ k-j+1 \leq i \leq k+j & \text { for even } j \leq k-1 \\ 2 \leq i \leq 2 k & \text { for } j=k \\ j-k-1 \leq i \leq 3 k-j & \text { for odd } j \geq k+2 \\ j-k \leq i \leq 3 k-j+1 & \text { for even } j \geq k+1\end{cases}
$$

If $k$ is even, then

$$
A_{i j}=1 \text { iff } \begin{cases}k-j+1 \leq i \leq k+j & \text { for odd } j \leq k-1 \\ k-j \leq i \leq k+j-1 & \text { for even } j \leq k-2 \\ 1 \leq i \leq 2 k-1 & \text { for } j=k \\ j-k \leq i \leq 3 k-j+1 & \text { for odd } j \geq k+1 \\ j-k+1 \leq i \leq 3 k-j+2 & \text { for even } j \geq k+2 .\end{cases}
$$

In both cases $A$ is symmetric.

Note also that $A$ is irreducible since its $(k+1)$ th row and column contain only ones.

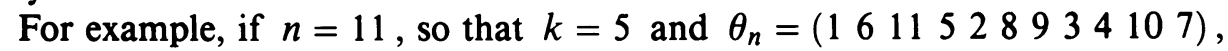
we have

$$
A=\left(\begin{array}{llllllllll}
0 & 0 & 0 & 0 & 0 & 1 & 1 & 0 & 0 & 0 \\
0 & 0 & 0 & 1 & 1 & 1 & 1 & 0 & 0 & 0 \\
0 & 0 & 0 & 1 & 1 & 1 & 1 & 1 & 1 & 0 \\
0 & 1 & 1 & 1 & 1 & 1 & 1 & 1 & 1 & 0 \\
0 & 1 & 1 & 1 & 1 & 1 & 1 & 1 & 1 & 1 \\
1 & 1 & 1 & 1 & 1 & 1 & 1 & 1 & 1 & 1 \\
1 & 1 & 1 & 1 & 1 & 1 & 1 & 1 & 0 & 0 \\
0 & 0 & 1 & 1 & 1 & 1 & 1 & 1 & 0 & 0 \\
0 & 0 & 1 & 1 & 1 & 1 & 0 & 0 & 0 & 0 \\
0 & 0 & 0 & 0 & 1 & 1 & 0 & 0 & 0 & 0
\end{array}\right)
$$


and

$$
A^{2}=\left(\begin{array}{llllllllll}
2 & 2 & 2 & 2 & 2 & 2 & 2 & 2 & 1 & 1 \\
2 & 4 & 4 & 4 & 4 & 4 & 4 & 4 & 3 & 2 \\
2 & 4 & 6 & 6 & 6 & 6 & 5 & 5 & 3 & 2 \\
2 & 4 & 6 & 8 & 8 & 8 & 7 & 6 & 4 & 2 \\
2 & 4 & 6 & 8 & 9 & 9 & 7 & 6 & 4 & 2 \\
2 & 4 & 6 & 8 & 9 & 10 & 8 & 6 & 4 & 2 \\
2 & 4 & 5 & 7 & 7 & 8 & 8 & 6 & 4 & 2 \\
2 & 4 & 5 & 6 & 6 & 6 & 6 & 6 & 4 & 2 \\
1 & 3 & 3 & 4 & 4 & 4 & 4 & 4 & 4 & 2 \\
1 & 2 & 2 & 2 & 2 & 2 & 2 & 2 & 2 & 2
\end{array}\right)
$$

Proof of Lemma 1. We describe here the case where $n \equiv 3(\bmod 4)$, i.e., $k=(n-1) / 2$ is odd. The case when $k$ is even is handled similarly.

Let $r=\left(u_{1}, \ldots, u_{k-1}, u_{k}, v_{k}, v_{k-1}, \ldots, v_{1}\right)$ be a (left) Perron-Frobenius eigenvector for $A=M\left(\theta_{n}\right)$, so in particular we have that $r$ is (strictly) positive and $r A^{2}=\lambda^{2} r$ with $\lambda$ the spectral radius of $A$. We use this, together with Lemma 2 and the fact that $r \in \mathscr{P}$.

Note first that $v_{1}-u_{1}=\lambda^{2}\left(v_{2}+v_{1}-u_{1}\right) \geq \lambda^{2} v_{1}>0$, and of course $u_{1}>0$. To verify that the inequalities in the first part of Definition 2 are in fact strict for $r$, we first observe that for $j=1,2, \ldots,(k-1) / 2$, we have $v_{2 j+1}>u_{2 j+1}$ since $v_{2 j+1}-u_{2 j+1} \geq v_{1}-u_{1}>0$, and similarly $u_{2 j}-v_{2 j} \geq v_{1}-u_{1}>0$. To see that for such $j, v_{2 j}>v_{2 j-1}$ and $u_{2 j+1}>u_{2 j}$, it suffices to notice that the $p$ th column of $A^{2}$ dominates the $(p-1)$ th (respectively $(p+1)$ th) column for $p \leq k$ (respectively $p>k$ ) and has some entries strictly larger.

It remains to check that the inequalities in the second part of the definition of $\mathscr{P}$ are strict for $r$. We consider half of these inequalities at a time. For $j=1,2, \ldots,(k-3) / 2$, we have

$$
\begin{aligned}
\left(v_{2 j+1}-u_{2 j+1}\right)-\left(u_{2 j}-v_{2 j}\right) & =v_{2 j}+v_{2 j+1}-\left(u_{2 j}+u_{2 j+1}\right) \\
& =\lambda^{2}\left(2 v_{2 j}+v_{2 j+1}+v_{2 j+2}-u_{2 j-1}-u_{2 j}-2 u_{2 j+1}\right) \\
& \geq \lambda^{2}\left(2 v_{2 j}-u_{2 j-1}-u_{2 j}\right) \\
& \geq \lambda^{4}\left(3 v_{2 j}-2 u_{2 j-1}\right) \\
& >0 .
\end{aligned}
$$

Also,

$$
\begin{aligned}
v_{k-1}+v_{k}-\left(u_{k-1}+u_{k}\right) & =\lambda^{2}\left(2 v_{k-1}+v_{k}-u_{k-2}-u_{k-1}-u_{k}\right) \\
& \geq \lambda^{2}\left(2 v_{k-1}-u_{k-2}-u_{k-1}\right) \\
& \geq \lambda^{4}\left(3 v_{k-1}-2 u_{k-2}\right) \\
& >0
\end{aligned}
$$

Similarly, for $j=2,3, \ldots,(k-1) / 2$, we have

$$
\begin{aligned}
v_{2 j-1}+u_{2 j}-\left(v_{2 j-1}+v_{2 j}\right) & =\lambda^{2}\left(2 u_{2 j-1}+u_{2 j}+u_{2 j+1}-v_{2 j-2}-v_{2 j-1}-2 v_{2 j}\right) \\
& \geq \lambda^{2}\left(2 u_{2 j-1}-v_{2 j-2}-v_{2 j-1}\right) \\
& \geq \lambda^{4}\left(3 u_{2 j-1}-2 v_{2 j-2}\right) \\
& >0
\end{aligned}
$$


and

$$
\begin{aligned}
u_{1}+u_{2}-\left(v_{1}+v_{2}\right) & =\lambda^{2}\left(2 u_{1}+u_{2}+u_{3}-v_{1}-2 v_{2}\right) \\
& \geq \lambda^{4}\left(3 u_{1}\right) \\
& >0
\end{aligned}
$$

\section{REFERENCES}

[Be] C. Bernhardt, The ordering on permutations induced by continuous maps of the real line, Ergodic Theory Dynamical Systems 7 (1987), 155-160.

[BCop] L. Block and W. A. Coppel, Dynamics in one dimension, Lecture Notes in Math., vol. 1513, Springer-Verlag, Berlin and New York, 1992.

[BGMY] L. Block, J. Guckenheimer, M. Misiurewicz, and L. S. Young, Periodic points and topological entropy of one-dimensional maps, Lecture Notes in Math., vol. 819, Springer-Verlag, Berlin and New York, 1980, pp. 18-34.

[GT] W. Geller and J. Tolosa, Maximal entropy odd orbit types, Trans. Amer. Math. Soc. 329 (1992), 161-172.

[J] I. Jungreis, Some results on the Sarkovskii partial ordering of permutations, Trans. Amer. Math. Soc. 325 (1991), 319.

[MN] M. Misiurewicz and Z. Nitecki, Combinatorial patterns for maps of the interval, Mem. Amer. Math. Soc., vol. 94, no. 456, Amer. Math. Soc., Providence, RI, 1991.

Mathematical Sciences Research Institute, 1000 Centennial Drive, Berkeley, CaliFORNIA 94720

Current address: Department of Mathematics, Indiana University-Purdue University Indianapolis, 402 N. Blackford St., Indianapolis, Indiana 46202

E-mail address: wgeller@math.iupui.edu

Institute of Mathematics, The Hebrew University of Jerusalem, Jerusalem, IsRael

E-mail address: weiss@sunrise.huji.ac.il 